\title{
Damage Detection in Railway Truss Bridges Employing Data Sensitivity under Bayesian Framework: A Numerical Investigation
}

\author{
Kanta Prajapat and Samit Ray-Chaudhuri \\ Department of Civil Engineering, IIT Kanpur, Kanpur 208016, India \\ Correspondence should be addressed to Samit Ray-Chaudhuri; samitrc@iitk.ac.in
}

Received 9 October 2016; Revised 23 February 2017; Accepted 12 March 2017; Published 6 April 2017

Academic Editor: Evgeny Petrov

Copyright (c) 2017 Kanta Prajapat and Samit Ray-Chaudhuri. This is an open access article distributed under the Creative Commons Attribution License, which permits unrestricted use, distribution, and reproduction in any medium, provided the original work is properly cited.

\begin{abstract}
In general, for a structure it is quite difficult to get information about all of its modes through its dynamic response under ambient or external excitation. Therefore, it is vital to exhaustively use the available information in the acquired modal data to detect any damage in the structures. Further, in a Bayesian algorithm, it can be quite beneficial if a damage localization algorithm is first used to localize damage in the structure. In this way, the number of unknown parameters in the Bayesian algorithm can be reduced significantly and thus, the efficiency of Bayesian algorithm can be enhanced. This study exploits a mode shape and its derivative based approach to localize damage in truss type structures. For damage quantification purpose, a parameter sensitivity based prediction error variance approach in Bayesian model updating is employed, which allows extracting maximum information available in the modal data. This work employs the sensitivity based Bayesian algorithm to determine the posterior confidence in truss type railway bridges. Results of the study show that the proposed approach can efficiently detect and quantify damage in railway truss bridges.
\end{abstract}

\section{Introduction}

The collapse or damage, especially in large structures such as bridges, can lead to huge losses in terms of human lives and economic burden. The repair after a collapse is a tedious and time taking task, which renders the bridge inoperable (downtime) for a considerable period of time adding to the indirect loss. Thus, all the major bridges are needed to be monitored in regular basis and needed to be repaired or rehabilitated whenever required. As specified in a recent report on the condition of Indian railway bridges by Indian Institute of Science (IISc), Bangalore (condition assessment of railway bridges, 2010, http://civil.iisc.ernet.in/crbridge.pdf), the damage has been induced in many of these bridges because of increase in average traffic and associated traffic loads. In fact, the design axle load has been increased from $18 \mathrm{t}$ to $22 \mathrm{t}$ in recent years. These factors may lead to minor damage such as cracks, which can be worrisome to railway authority.
In the last few decades, Bayesian model updating has rapidly arisen as a reliable and effective approach to solve system identification problems probabilistically and, in turn, to detect damage in a system. The efficiency of Bayesian model updating depends on various issues such as the efficiency of simulation algorithm, data used for updating, prior distributions, and likelihood function. Many of these issues have been successfully resolved in recent years [1-13]. Any kind of damage in a structure causes change in its structural parameters, for example, stiffness and mass. Subsequently, the dynamic properties such as natural frequencies and mode shapes also get changed. The change in these dynamic properties due to a change in structural parameters has been utilized for years to identify damage in structures. Sohn and Law [14] employed incomplete and noisy modal data to localize and quantify the amount of damage under Bayesian inference. A damage localization technique in structures under Bayesian inference using modal data is presented by Huhtala and 
Bossuyt [15] for a steel cantilever beam. Damage detection in plate type structures is studied by Kurata et al. [16]. Simoen et al. [17] did damage assessment of a slice of 7-story RC building using Bayesian uncertainty quantification technique.

The efficiency of any Bayesian quantification algorithm can be improved significantly by reducing the number of unknowns in the problem. This can be achieved by using a damage localization algorithm to localize damage in the structure before quantifying the damage in the structure. The Bayesian algorithm basically works on error minimization between the response of a mathematical model and the response of the actual structure to update the parameters of the mathematical model. This is achieved in repeated cycles or repeated runs of Markov chain. In one run of Markov chain, each unknown parameter is picked up one by one and its new proposed value is being compared to accept or reject with the previous value for a better fit of the actual structural response. While doing this, all other unknown parameters are being kept on the best achieved values from the previous or current run, which are not the actual value of these parameters. Therefore, when an inverse problem is solved under Bayesian inference with many unknown parameters and limited Bayesian evidence, the convergence or getting the stationary distribution of Markov chain becomes difficult. It costs both the computational efficiency and the accuracy of the solution. This is particularly true when the employed data in Bayesian evidence are contaminated with noise, which is a general situation to find in any practical application. The present noise in the data may lead to the convergence of the chain to a false solution. By employing the information about the undamaged members using the damage localization algorithm, the Bayesian algorithm is made to search the optimum value of unknown parameters in right direction. This causes the algorithm to escape any false solution of the inverse problem. Hence, the accuracy and computational efficiency of the Bayesian algorithm can be improved by limiting the number of unknown parameters with the localization algorithm.

It is evident from literature that comparison of mode shape and its derivatives for undamaged and damaged structures effectively localizes damage in a structure. Modes shapes curvatures idea for damage localization was first proposed by Pandey et al. [18] in which mode shape curvature was said to be sensitive parameter for damage localization. Sampaio et al. [19] extended the idea of Pandey et al. [18] by using the curvature-based method for frequency response function instead of mode shape and demonstrated the potential of this approach by considering real time data. Later, Wahab [20] utilized mode shape curvature along with natural frequencies and mode shapes for finite element model updating. Ray Chaudhuri [21] investigated the behaviour of eigenproperties, namely, frequencies and mode shapes, in presence of stiffness degradation in simulated structures such as shear building and steel moment-resisting frame (SMRF). A mathematical formulation with the use of perturbation approach was developed in this study to inspect the change in frequencies as well as mode shapes for a given location of stiffness degradation. It may be noted that higher modes are usually difficult to get for a typical civil engineering structure as these modes do not get excited under ambient conditions due to higher input energy requirements. Thus, the fundamental mode is more frequently used for damage detection. Zhu et al. [22] demonstrated the efficiency of the change in slope of the first mode shape as a damage sensitive feature by performing a numerical study on an eight-story shear building and conducting experiments on a three-story building model. Dilena et al. [23] demonstrated that mode shape curvature can be a useful term for damage location on a reinforced concrete single span bridge. Bai et al. [24] applied mode shape curvature-based technique on a two-dimensional plate grid structure to locate the damage in the structure. Recently, Roy and Ray-Chaudhuri [25] proposed a mathematical basis to establish a correlation between structural damage and change in the structure's fundamental mode shape and its derivatives using a perturbation approach. In this work, this approach is used to localize the damage in the truss type structure.

When modal data is used as evidence in a Bayesian damage detection algorithm, many studies have suggested different ways to take variance for prediction error model of frequency and mode shape data types [26-30]. Most of these studies consider only two variances: one for frequencies of all modes and the other for mode shape components of all modes. Only a few studies consider separate variances for data of different modes. However, depending on various conditions, all frequencies and mode shape components of all modes may require separate variances for the prediction error models for an efficient information extraction from these data points. This study employs a sensitivity based approach recently developed by the authors [30] to derive the variances for prediction error models of different data points to efficiently extract the information from these data points.

This work is structured in three parts: the first part deals with damage localization, whereas the second and third parts emphasize damage quantification and posterior resolution of unknown parameters, respectively, in a railway truss bridge. For localization purpose, change in the fundamental mode shape based algorithm is used. Quantification and posterior resolution are achieved employing the sensitivity based algorithm under Bayesian inference. The modal data from the first two modes are used for updating the stiffness parameters of the truss bridge model. Markov Chain Monte Carlo simulation technique is employed using MetropolisHasting algorithm to simulate the samples from the posterior distribution. The mean of the posterior distribution is taken as the parameter estimation of distribution to represent the unknown stiffness parameters. Results of the study show the efficiency of used approaches for damage detection in truss type bridges.

\section{Mode Shape Based Damage Localization}

From literature it is evident that the damage in a structure can be localized by comparing its damaged and undamaged mode shapes and their derivatives. Recently, Roy and RayChaudhuri [25] proposed a mathematical basis to establish a correlation between structural damage and change in the structure's fundamental mode shape and its derivatives using a perturbation approach. 


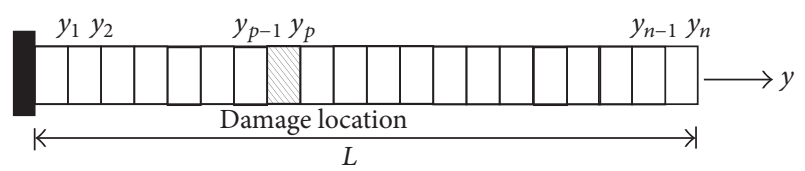

FIgURE 1: Schematic diagram of a shear beam.

For a shear beam (Figure 1), they have illustrated that the difference between the damaged and undamaged fundamental mode shapes can be expressed as

$$
\partial \phi\left(y_{l}\right)=\left\{\begin{array}{lll}
\mu\left[\frac{\pi}{2}-f\left(y_{l}\right)\right], & y_{l}>y_{p} & \\
\mu\left[\frac{\pi}{4}-f\left(y_{l}\right)\right], & y_{l}=y_{p} & l=1, \ldots, n ; \\
\mu\left[-f\left(y_{l}\right)\right], & y_{l}<y_{p} ;
\end{array}\right.
$$

here $\mu$ is a constant as given in Roy and Ray-Chaudhuri [25] and $f\left(y_{l}\right)$ is as follows:

$$
f\left(y_{l}\right)=2 \sin \left(\frac{\pi y_{l}}{2 L}\right) \cos \left(\frac{\pi y_{p}}{2 L}\right) .
$$

A jump of $\mu \pi / 2$ can be observed in the function $\partial \phi\left(y_{l}\right)$ between the locations $y_{l}=y_{p-1}$ and $y_{l}=y_{p+1}$ (1). This causes a steep slope $\partial \phi^{\prime}\left(y_{l}\right)$ around $y_{l}=y_{p}$ (location of damage). It may also be observed that if the beam is discretized into a large number of elements, that is, $n \rightarrow \infty$, this slope approaches a Dirac delta-type function. Further, this implies that the second derivative of $\partial \phi\left(y_{l}\right)$, that is, $\partial \phi^{\prime \prime}\left(y_{l}\right)$, changes its sign at $y_{l}=y_{p}$. Therefore, by looking at the difference of damaged and undamaged mode shape and the first and second derivatives of this difference, the location of the damage in a structure can be determined.

This mode shape based approach is adopted to localize damage in truss structures. For this purpose, a twodimensional numerical model for a truss type structure is developed in an open source finite element software, OpenSees (http://opensees.berkeley.edu/). The modal data in the healthy state of the structure are referred to as undamaged data of the structure. To simulate damage in a truss panel, the stiffness of the considered member is reduced by $50 \%$.

Figures 2-5 show the plot of mode shape difference and its derivatives for different damage locations. For vertical web members, a peak in mode shape difference and its curvature is observed, whereas the slope of the difference shows a zero crossing at the location of damage. These signature patterns can be used to localize damage in the truss structure. It is to be noted here that, for Figure 2, the fundamental mode shape corresponding to the bottom chord is used, whereas, in Figure 3, the fundamental mode shape corresponding to the top chord is utilized for damage localization. For member V6, the bottom chord fundamental mode shape is unable to identify the damage efficiently. Therefore, the top chord mode shape has been used. A slight change in pattern of these signatures can also be observed for damage in vertical, inclined, and bottom chord members (Figures 2-5). This is because the effective damaged degrees of freedom are different in these cases. If a structure is not damaged in multiple locations, then the mode shape based approach can be effectively used to localize damage in the structure. This localization information further can be used in Bayesian algorithm to quantify damage in the structure in an efficient way. Next section presents a Bayesian damage quantification algorithm applied to a truss type structure. To see the efficiency of quantification algorithm without using any localization algorithm, all bottom chords members' stiffness is assumed to be unknown. However, in practice the result of localization algorithm can be used before quantification of damage.

\section{Bayesian Model Updating with Modal Data}

To predict the response of a physical system due to a future excitation, a correct mathematical model for that system is required. A mathematical formulation for a physical problem involves the understanding of the basic physical laws associated with the problem, associated material properties, and then representing those in the language of mathematics. However, a true behaviour of a structural system can only be understood by studying its response under applied loads. In Bayesian model updating, the parameters of a system that define its mathematical model are updated using the response of the system itself so that a more reliable mathematical model of the system can be achieved. The updating is assumed to be satisfactory when the response of the mathematical model matches with the response of the physical system for a given input.

In Bayesian model updating this is done using Bayes' theorem as given below:

$$
p(\boldsymbol{\theta} \mid \mathbf{D})=\frac{p(\mathbf{D} \mid \boldsymbol{\theta}) p(\boldsymbol{\theta})}{p(\mathbf{D})},
$$

where $\boldsymbol{\theta} \in R^{n}$ is the parameter vector, which needs to be updated and $\mathbf{D}$ is the available evidence from the system. Expression $p(\boldsymbol{\theta})$ is known as the prior distribution of parameter vector $\boldsymbol{\theta}$ and $p(\mathbf{D} \mid \boldsymbol{\theta})$ represents the probability of the evidence $\mathbf{D}$ when a belief of $\boldsymbol{\theta}$ is taken as true. The term $p(\mathbf{D} \mid \boldsymbol{\theta})$ is called the likelihood of the evidence for that belief. The total probability of the evidence $\mathbf{D}$ for the model is a constant. This can be given by the sum of the likelihood of the evidence for each and every belief of $\boldsymbol{\theta}$, that is, $\sum_{i=1}^{n} p(\mathbf{D}$ | $\left.\boldsymbol{\theta}_{i}\right)$ and is represented as $p(\mathbf{D})$. The expression $p(\boldsymbol{\theta} \mid \mathbf{D})$ is called the posterior distribution of the parameter vector $\boldsymbol{\theta}$. If the evidence $\mathbf{D}$ consists of modal data of the system, it can be shown that likelihood for frequency and mode shape components can be expressed as

$$
\begin{aligned}
& p\left(\frac{\omega_{i}}{\boldsymbol{\theta}}, \sigma_{\omega_{i}}\right)=\frac{1}{\sigma_{\omega_{i}} \sqrt{2 \pi}} e^{-\left(\omega_{i}-\widehat{\omega}_{i}(\boldsymbol{\theta})\right)^{2} / 2 \sigma_{\omega_{i}}{ }^{2}} . \\
& p\left(\frac{\boldsymbol{\varphi}_{i}}{\boldsymbol{\theta}}, \mathbf{V}_{\boldsymbol{\varphi}_{i}}\right)=\frac{\mathbf{V}_{\boldsymbol{\varphi}_{i}}^{-1 / 2}}{(2 \pi)^{n / 2}} \\
& \cdot \exp \left(-\frac{1}{2}\left(\boldsymbol{\varphi}_{i}-\widehat{\boldsymbol{\varphi}}_{i}(\boldsymbol{\theta})\right)^{T} \mathbf{V}_{\boldsymbol{\varphi}_{i}}{ }^{-1}\left(\boldsymbol{\varphi}_{i}-\widehat{\boldsymbol{\varphi}}_{i}(\boldsymbol{\theta})\right)\right) .
\end{aligned}
$$



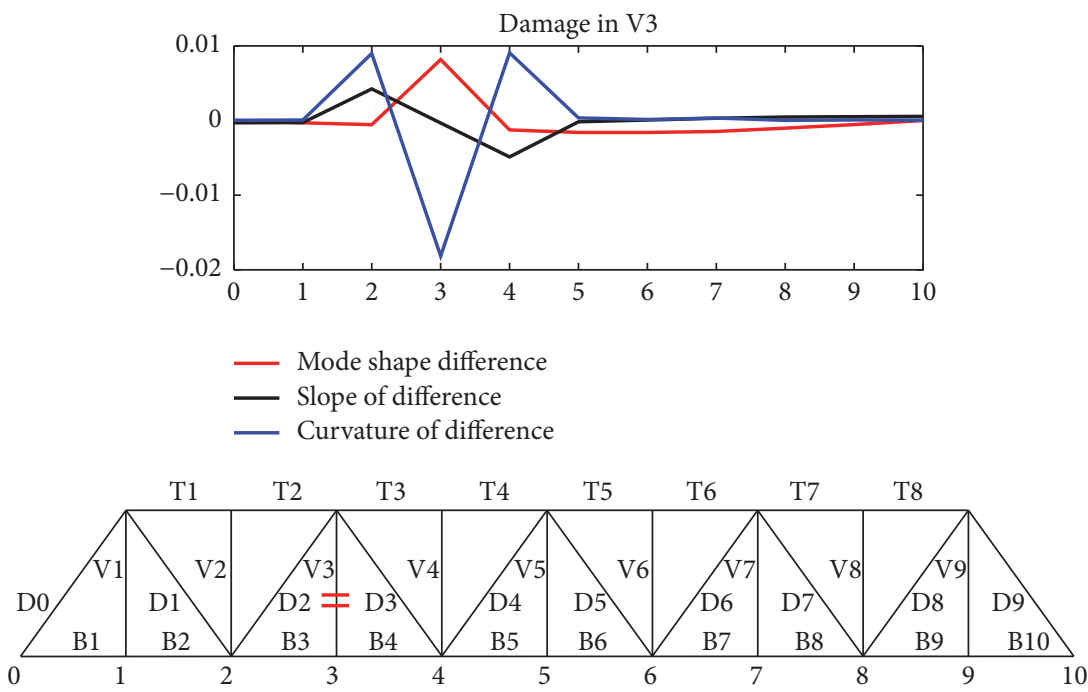

FIgURE 2: Damage localization for damage location V3.

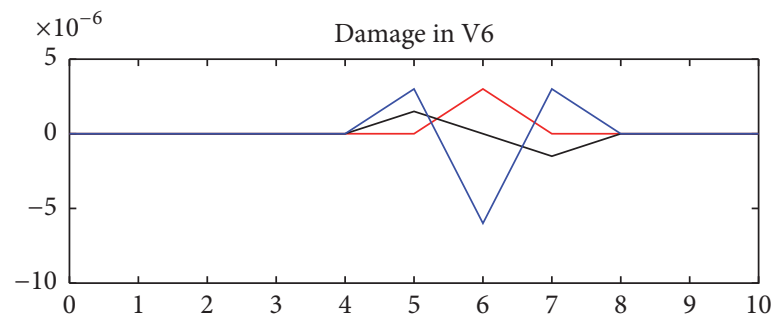

- Mode shape difference

- Slope of difference

— Curvature of difference

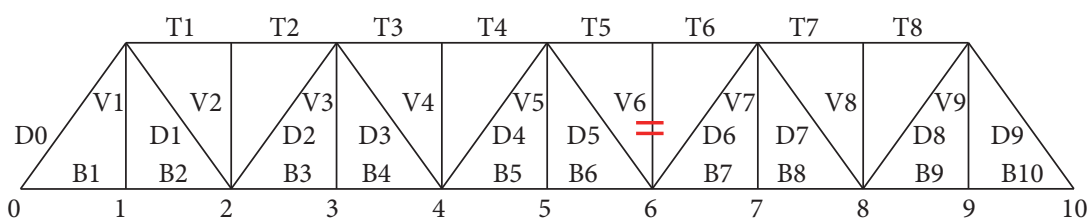

FIgURE 3: Damage localization for damage location V6.

Here, $\omega_{i}$ and $\boldsymbol{\varphi}_{i}$ represent the observed frequency and the mode shape vector of the $i$ th mode of the system $i=1, \ldots, m$. Frequency and mode shape of the mathematical model for the $i$ th mode are represented as $\widehat{\omega}_{i}(\boldsymbol{\theta})$ and $\widehat{\varphi}_{i}(\boldsymbol{\theta})$, respectively. The likelihood formation for frequency and mode shape components is done assuming that the deviation between the system and model output is normally distributed. In other words, the prediction error models for the used data points are assumed to be normally distributed with some standard deviation or variance. Standard deviation of the deviation in frequency of the $i$ th mode is taken as $\sigma_{\omega_{i}}$. Here, $\mathbf{V}_{\boldsymbol{\varphi}_{i}}$ represents the covariance matrix of the deviation in $i$ th mode shape vector components. Further, it is assumed that the frequency and mode shape of an energy mode are statically independent, informatively. Now, if each mode is also assumed independent of other modes then for $m$ modes the likelihood of the evidence $\mathbf{D}$ can be given as

$$
p\left(\frac{\mathbf{D}}{\boldsymbol{\theta}}\right)=\prod_{i=1}^{m} p\left(\omega_{i} \mid \boldsymbol{\theta}, \sigma_{\omega_{i}}\right) \cdot p\left(\boldsymbol{\varphi}_{i} \mid \boldsymbol{\theta}, \mathbf{V}_{\boldsymbol{\varphi}_{i}}\right) .
$$

To evaluate the covariance matrix $\mathbf{V}_{\varphi_{i}}$ in above equation, mode shape components are taken as uncorrelated to each other. As a result, $\mathbf{V}_{\boldsymbol{\varphi}_{i}}$ comes to be a diagonal matrix. Now, if $\boldsymbol{d}$ is the length of the parameter vector $\boldsymbol{\theta}$, then the total unknown parameters in updating problem increase to $d+$ $m(n+1)$, where $n$ is the number of observed degrees of freedom. The unknown parameter vector $\boldsymbol{\theta}$ thus becomes

$$
\overline{\boldsymbol{\theta}}=\left\{\boldsymbol{\theta}^{T},\left\{\sigma_{\omega_{i}}^{2}, \mathbf{V}_{\boldsymbol{\varphi}_{i} 11}, \ldots, \mathbf{V}_{\boldsymbol{\varphi}_{i} n n}\right\}_{i=1}^{m}\right\}^{T} .
$$



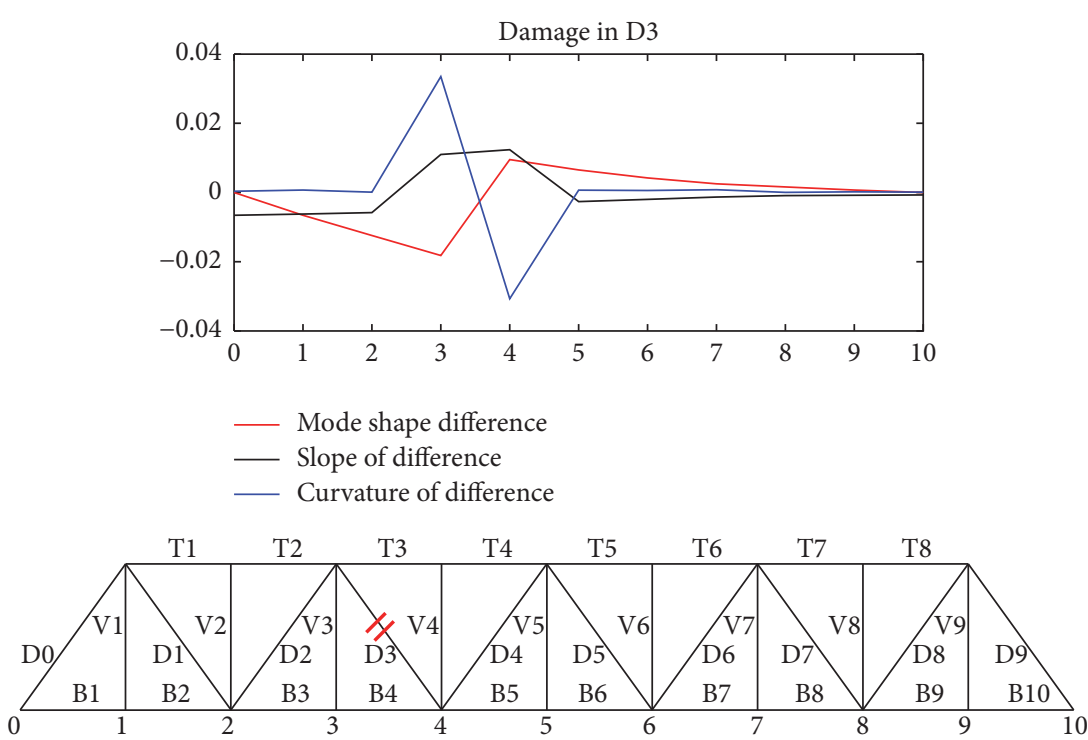

Figure 4: Damage localization for damage location D3.

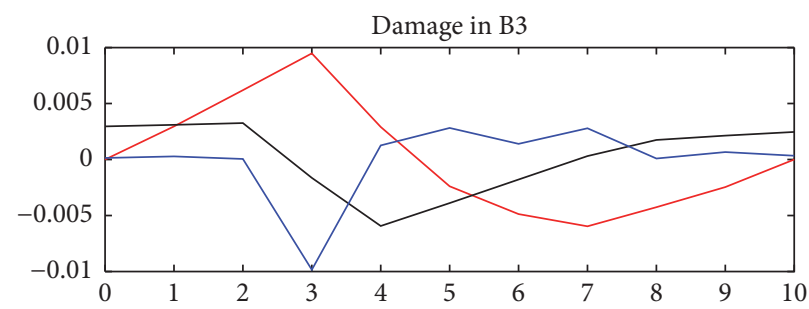

- Mode shape difference

- Slope of difference

_ Curvature of difference

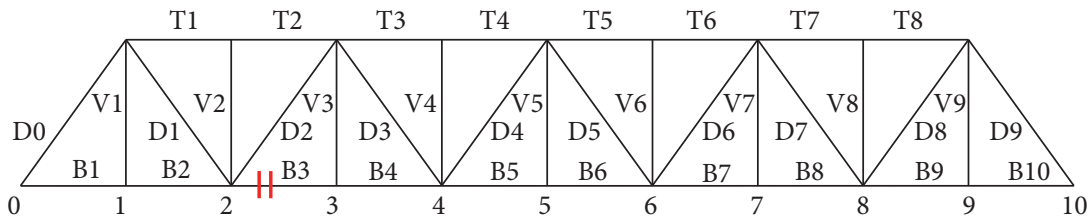

Figure 5: Damage localization for damage location B3.

Equation (5) now can be rewritten as

$$
p\left(\frac{\mathbf{D}}{\overline{\boldsymbol{\theta}}}\right)=\prod_{i=1}^{m} p\left(\omega_{i} \mid \overline{\boldsymbol{\theta}}\right) \prod_{j=1}^{n} p\left(\boldsymbol{\varphi}_{i j} \mid \overline{\boldsymbol{\theta}}\right) .
$$

In a recent study by the authors [30], it is shown that the sensitivity of modal data towards structural parameters can be used to evaluate the normalized variances of error models of frequency and mode shape components of different modes. In this way, exhaustive information from the data used for the updating can be obtained without increasing the number of unknown parameters (unknown variances for each data point) in the updating algorithm. The present study uses this approach to evaluate the unknown variances.

\section{Numerical Modelling}

For numerical investigation, 2D model for a typical railway bridge is prepared in a finite element software package OpenSees (http://opensees.berkeley.edu/). Figure 6 shows the schematic diagram of the 2D model developed in OpenSees. Dynamic properties of the models are evaluated by performing modal analysis in OpenSees.

To illustrate the effectiveness of the sensitivity based algorithm in damage detection of truss type structures, stiffness of some members are degraded in the truss model by some known amount. Then the modal data from this damaged structure is treated as the evidence in the algorithm. The algorithm further treats the stiffness of the truss member as unknown. The stiffness of these unknown members is then evaluated using the algorithm. 


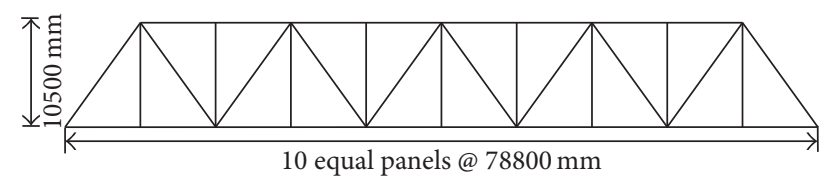

FIGURE 6: Schematic diagram of truss geometry for a typical railway bridge.

TABLE 1: Posterior statistics obtained using proposed approach for 2D full scale model.

\begin{tabular}{lccc}
\hline $\begin{array}{l}\text { Unknown parameters } \\
\text { with actual value }\end{array}$ & Mean & Variance & $\begin{array}{c}\text { Absolute } \\
\text { deviation (\%) }\end{array}$ \\
\hline$k_{1}=0.5$ & 0.48 & 0 & 3.66 \\
$k_{2}=0.7$ & 0.70 & 0.0001 & 0.60 \\
$k_{3}=0.5$ & 0.48 & 0.0002 & 3.42 \\
$k_{4}=0.9$ & 0.87 & 0.0003 & 3.52 \\
$k_{5}=0.6$ & 0.55 & 0.0044 & 9.07 \\
$k_{6}=1.0$ & 1.31 & 0.1025 & 31.02 \\
$k_{7}=0.8$ & 0.75 & 0.0004 & 6.46 \\
$k_{8}=0.4$ & 0.42 & 0.0006 & 5.37 \\
$k_{9}=0.5$ & 0.47 & 0.0005 & 5.08 \\
$k_{10}=0.6$ & 0.59 & 0.001 & 2.10 \\
\hline
\end{tabular}

For the purpose of numerical illustration, the bottom chord members of the $2 \mathrm{D}$ model have been degraded in stiffness with some known amount. Data (frequencies and mode shapes) from the first two modes of this damaged structure are considered as the evidence. This evidence data is further contaminated by a noise of coefficient of variation $5 \%$ to represent the actual practical scenario of updating scheme. A total of 15 such contaminated data sets are then taken to find the stiffness of unknown bottom chord members. All other structural parameters are taken as known in the algorithm.

To avoid any biasness in the algorithm, exponential prior with mean value of 2 which is very far from the actual adopted value of stiffness factors $k_{i}, i=1$ to 10 (10 panels of bottom chord), is adopted for the choice of prior distribution of all unknown stiffness parameters. The total unknown parameters contain the unknown stiffness parameters, multiplication factor of normalized variances, and shape parameter of proposal distribution of this multiplication factor. Prior distribution of these additional two unknown parameters is taken as uniform in a range 0.00001 to 10 for multiplication factor and 1 to 1000000 for shape parameter. Gamma distribution is adopted as the proposal distribution for all the unknown parameters. MetropolisHasting Markov Chain Monte Carlo (MCMC) simulation algorithm is employed to draw samples from the high dimensional posterior distribution.

Table 1 shows the results obtained using proposed algorithm for the two-dimensional model. Results are presented in terms of mean and variance of the posterior distribution. It can be observed from Table 1 that the proposed algorithm is efficient to obtain unknown parameters using only first two modes' data as evidence. The maximum deviation obtained is $31.02 \%$. Figure 7 shows the posterior distribution of the parameters. It is evident from Figure 7 that posterior distribution is very concentrated near the actual values of parameters for most of the unknown parameters in spite of a large range prior distribution for the parameters. The Markov chain of run for all the unknown stiffness parameters is shown in Figure 8. It can be observed form Figure 8 that, except a few parameters, the Markov chain reaches its stationary distribution after approximately 100 runs of the simulation. The convergence of Markov chain can be taken as a good estimate of accuracy of unknown parameters. However, it is evident in some cases that, sometimes due to the present noise levels in the evidence data or due to the insensitivity of modal data to certain unknown parameters, the Markov chain may converge to a false value of unknown parameter. Therefore, a more robust and logical reasoning is required for the posterior resolution of the unknown parameters. The subsequent section describes a sensitivity based posterior resolution algorithm and its application for the present numerical example.

\section{Posterior Resolution}

This section presents a sensitivity based approach for the posterior resolution of unknown parameters. The algorithm is originally given by Prajapat and Ray-Chaudhuri [31] and briefly regenerated here. Any Bayesian model updating algorithm works on the error minimization between response of the system and the response of the mathematical model defined with some parameters. Therefore, uncertainty in the value of an unknown parameter can be assumed to be inversely proportional to the ability of that parameter to change the response of the model with a change in the parameter itself. This ability of a parameter can be defined with a term "parameter impact" $\left(\mu_{k}\right)$, which is defined as the absolute sum of the first-order derivative of each and every modal data used for updating with respect to that parameter. Expression for "parameter impact" $\left(\mu_{k}\right)$ can be written as

$$
\begin{aligned}
\mu_{k_{i}}=\left|\frac{\partial \omega_{I}^{2}}{\partial k_{i}}\right|+\left|\frac{\partial \varphi_{I^{j}}}{\partial k_{i}}\right| & ; \\
I & =1, \ldots, m j=1, \ldots, n i=1, \ldots, t,
\end{aligned}
$$

where $m$ is the number of considered modes, $n$ is the number of observed degrees of freedom, and $t$ is the total number of unknown parameters. Therefore, the uncertainty $\psi_{k_{i}}$ in parameter $k_{i}$ can be given as [31]

$$
\psi_{k_{i}} \propto \frac{1}{\mu_{k_{i}}} .
$$

The above relation is used for the meaningful posterior resolution of the unknown parameters in the present numerical example of the truss structure. Table 2 shows the normalized parameter impact $\mu_{k_{i}}^{n}$ (normalized with the maximum $\mu_{k_{i}}$ ) for each unknown parameter. It can be observed from Table 2 that the maximum deviation is for the parameter which has the lowest normalized parameter 


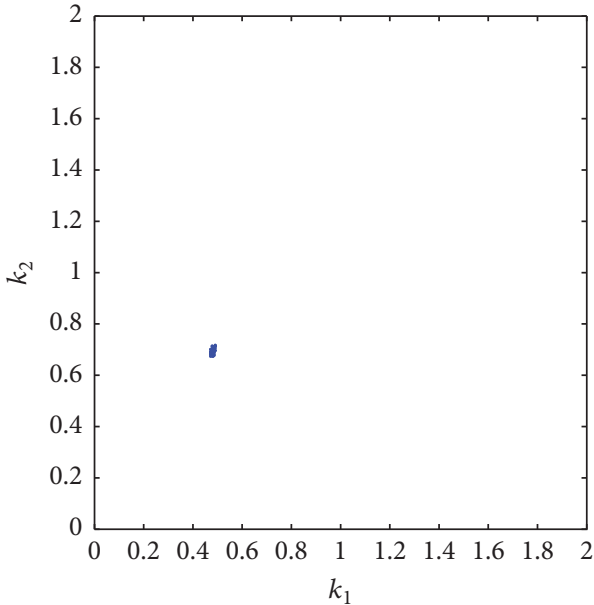

(a)

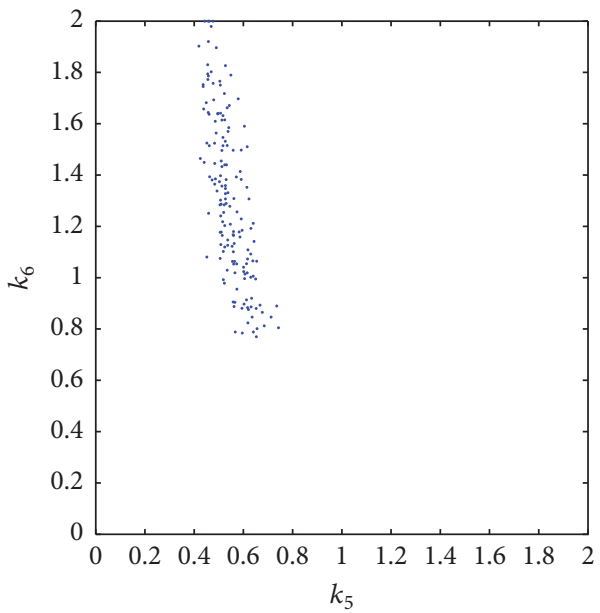

(c)

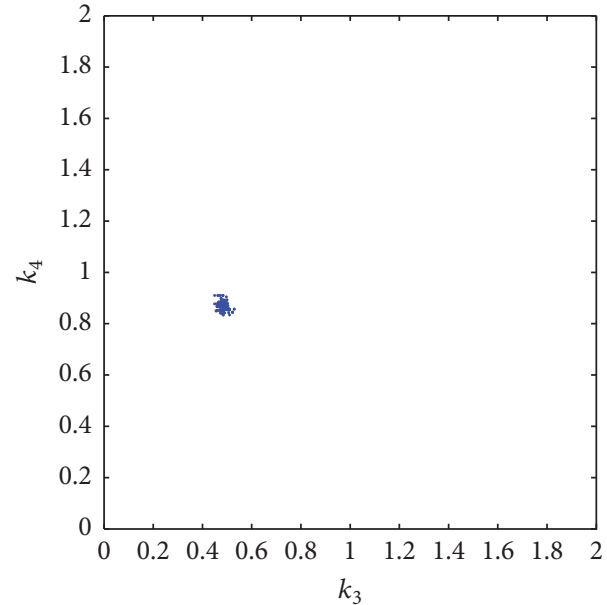

(b)

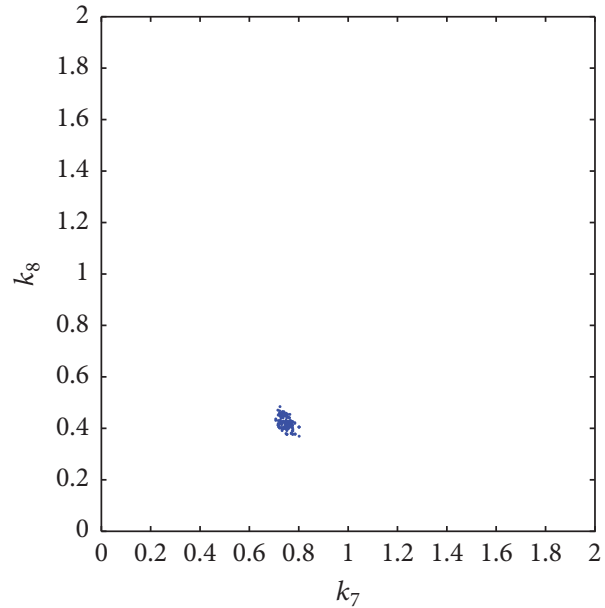

(d)

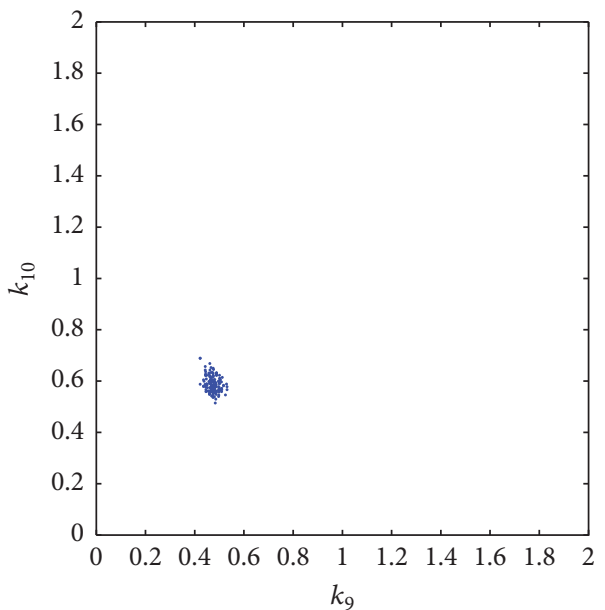

(e)

FIGURE 7: Posterior distribution of unknown stiffness parameters. 


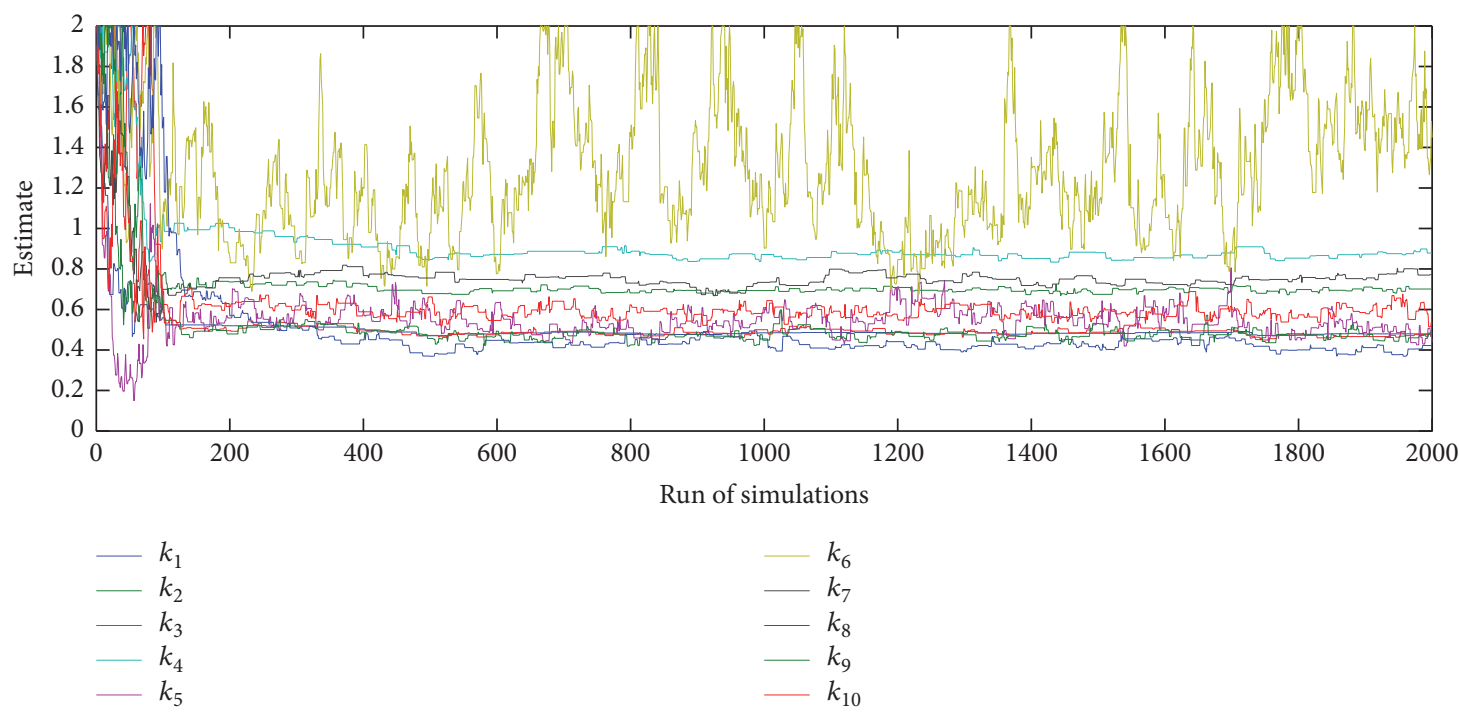

FIGURE 8: Markov chain for different unknown stiffness parameters.

TABLE 2: Normalized parameter impact for different unknown parameters.

\begin{tabular}{lcc}
\hline $\begin{array}{l}\text { Unknown parameters } \\
\text { with actual value }\end{array}$ & $\begin{array}{c}\text { Normalized } \\
\text { parameter impact } \\
\left(\mu_{k_{i}}^{n}\right)\end{array}$ & $\begin{array}{c}\text { Absolute deviation } \\
(\%)\end{array}$ \\
\hline$k_{1}=0.5$ & 1 & 3.66 \\
$k_{2}=0.7$ & 0.44 & 0.60 \\
$k_{3}=0.5$ & 0.48 & 3.42 \\
$k_{4}=0.9$ & 0.48 & 3.52 \\
$k_{5}=0.6$ & 0.17 & 9.07 \\
$k_{6}=1.0$ & 0.07 & 31.02 \\
$k_{7}=0.8$ & 0.39 & 6.46 \\
$k_{8}=0.4$ & 0.36 & 5.37 \\
$k_{9}=0.5$ & 0.25 & 5.08 \\
$k_{10}=0.6$ & 0.22 & 2.10 \\
\hline
\end{tabular}

impact. Therefore, it can be concluded that the algorithm successfully resolves the most uncertain and certain parameters in the truss. It can also be observed from Figure 7 that, for parameter $k_{6}$, the distribution is quite scattered compared to other parameters. The Markov chain for parameter $k_{6}$ also does not reach its stationary distribution (Figure 8). Although no specific trend is observed between the normalized parameter impact $\left(\mu_{k}^{n}\right)$ and deviation, a low parameter impact in general tends to be associated with a poorly resolved parameter. Although a higher parameter impact may not guarantee a best resolved parameter, a comparatively very low parameter impact guarantees a poorly resolved parameter. Authors believe that a more meaningful posterior resolution of unknown parameters can be obtained if different modes used for updating are considered separately for determining the parameter impact with appropriate measures. However, this is outside the scope of the present study.

\section{Conclusions}

Damage localization and quantification are done in truss type structures using some recently developed algorithms. For localization purpose, mode shape and its curvature-based approach is used. A recently developed sensitivity based Bayesian damage detection algorithm is employed for damage quantification in typical Indian railway truss type bridges. Along with the damage localization and quantification, the uncertainty in the resolved parameters is also determined. It is observed that the sensitivity based approach under Bayesian inference is capable of resolving the most uncertain unknown parameters in posterior statistics for these structures. It is also observed that the posterior variance and convergence of Markov chain can be used as a good measure of parameter accuracy in posterior statistics. However, in case of highly noisy data, the reliability of these approaches suffers. The results of the study show the efficiency of the approaches in damage localization and quantification of truss type structures.

\section{Conflicts of Interest}

The authors declare that they have no conflicts of interest.

\section{References}

[1] J. L. Beck and L. S. Katafygiotis, "Updating of a model and its uncertainties utilizing dynamic test data," in Computational Stochastic Mechanics, pp. 125-136, Springer, Berlin, Germany, 1991.

[2] J. L. Beck and L. S. Katafygiotis, "Updating models and their uncertainties. I: bayesian statistical framework," Journal of Engineering Mechanics, vol. 124, no. 4, pp. 455-461, 1998.

[3] J. L. Beck and S.-K. Au, "Bayesian updating of structural models and reliability using Markov chain Monte Carlo simulation," Journal of Engineering Mechanics, vol. 128, no. 4, pp. 380-391, 2002. 
[4] J. L. Beck and K.-V. Yuen, "Model selection using response measurements: Bayesian probabilistic approach," Journal of Engineering Mechanics, vol. 130, no. 2, pp. 192-203, 2004.

[5] I. Boulkaibet, T. Marwala, L. Mthembu, M. Friswell, and S. Adhikari, "Sampling techniques in Bayesian finite element model updating," https://arxiv.org/abs/1110.3382.

[6] L. Chen, Z. Qin, and J. S. Liu, "Exploring hybrid Monte Carlo in Bayesian computation," Sigma, vol. 2, pp. 2-5, 2001.

[7] S. H. Cheung and J. L. Beck, "Bayesian model updating using hybrid Monte Carlo simulation with application to structural dynamic models with many uncertain parameters," Journal of Engineering Mechanics, vol. 135, no. 4, pp. 243-255, 2009.

[8] S. H. Cheung and J. L. Beck, "Calculation of posterior probabilities for bayesian model class assessment and averaging from posterior samples based on dynamic system data," ComputerAided Civil and Infrastructure Engineering, vol. 25, no. 5, pp. 304-321, 2010.

[9] J. Ching, M. Muto, and J. L. Beck, "Bayesian linear structural model updating using gibbs sampler with modal data," in Proceedings of the 9th International Conference on Structural Safety and Reliability, pp. 2609-2616, Millpress, Rome, Italy, 2005.

[10] J. Ching and Y.-C. Chen, "Transitional Markov chain Monte Carlo method for Bayesian model updating, model class selection, and model averaging," Journal of Engineering Mechanics, vol. 133, no. 7, pp. 816-832, 2007.

[11] T. Marwala, L. Mdlazi, and S. Sibisi, "Finite element model updating using Bayesian approach," in Proceedings of the 23rd Conference and Exposition on Structural Dynamics (IMACXXIII '05), Orlando, Fla, USA, February 2005.

[12] L. Mthembu, T. Marwala, M. I. Friswell, and S. Adhikari, "Bayesian evidence for finite element model updating," https:// arxiv.org/abs/0810.2643.

[13] C. Papadimitriou, J. L. Beck, and L. S. Katafygiotis, "Updating robust reliability using structural test data," Probabilistic Engineering Mechanics, vol. 16, no. 2, pp. 103-113, 2001.

[14] H. Sohn and K. H. Law, "A Bayesian probabilistic approach for structure damage detection," Earthquake Engineering and Structural Dynamics, vol. 26, no. 12, pp. 1259-1281, 1997.

[15] A. Huhtala and S. Bossuyt, "A Bayesian approach to vibration based structural health monitoring with experimental verification," Journal of Structural Mechanics, vol. 44, no. 4, pp. 330344, 2011.

[16] M. Kurata, J. Lynch, K. Law, and L. Salvino, "Bayesian model updating approach for systematic damage detection of platetype structures," in Topics in Model Validation and Uncertainity Quantification, vol. 4, pp. 85-94, Springer, 2012.

[17] E. Simoen, B. Moaveni, J. P. Conte, and G. Lombaert, "Uncertainty quantification in the assessment of progressive damage in a 7-story full-scale building slice," Journal of Engineering Mechanics, vol. 139, no. 12, pp. 1818-1830, 2013.

[18] A. K. Pandey, M. Biswas, and M. M. Samman, "Damage detection from changes in curvature mode shapes," Journal of Sound and Vibration, vol. 145, no. 2, pp. 321-332, 1991.

[19] R. P. C. Sampaio, N. M. M. Maia, and J. M. M. Silva, "Damage detection using the frequency-response-function curvature method," Journal of Sound and Vibration, vol. 226, no. 5, pp. 1029-1042, 1999.

[20] M. M. A. Wahab, "Effect of modal curvatures on damage detection using model updating," Mechanical Systems and Signal Processing, vol. 15, no. 2, pp. 439-445, 2001.
[21] S. Ray Chaudhuri, "Change in instantaneous eigenproperties due to yielding of a structure," Journal of Sound and Vibration, vol. 312, no. 4-5, pp. 754-768, 2008.

[22] H. Zhu, L. Li, and X.-Q. He, "Damage detection method for shear buildings using the changes in the first mode shape slopes," Computers and Structures, vol. 89, no. 9-10, pp. 733-743, 2011.

[23] M. Dilena, A. Morassi, and M. Perin, "Dynamic identification of a reinforced concrete damaged bridge," Mechanical Systems and Signal Processing, vol. 25, no. 8, pp. 2990-3009, 2011.

[24] Y. Bai, S. He, W. Nie, J. Gao, and X. Song, "Plane grid structure damage location identification by model curvature," Procedia Engineering, vol. 31, pp. 534-540, 2012.

[25] K. Roy and S. Ray-Chaudhuri, "Fundamental mode shape and its derivatives in structural damage localization," Journal of Sound and Vibration, vol. 332, no. 21, pp. 5584-5593, 2013.

[26] I. Behmanesh, B. Moaveni, G. Lombaert, and C. Papadimitriou, "Hierarchical Bayesian model updating for structural identification," Mechanical Systems and Signal Processing, vol. 64-65, pp. 360-376, 2015.

[27] K. Christodoulou and C. Papadimitriou, "Structural identification based on optimally weighted modal residuals," Mechanical Systems and Signal Processing, vol. 21, no. 1, pp. 4-23, 2007.

[28] B. Goller and G. I. Schuëller, "Investigation of model uncertainties in Bayesian structural model updating," Journal of Sound and Vibration, vol. 330, no. 25, pp. 6122-6136, 2011.

[29] C. Papadimitriou, C. Argyris, D. C. Papadioti, and P. Panetsos, "Uncertainty calibration of large-order models of bridges using ambient vibration measurements," in Proceedings of the European Workshop on Structural Health Monitoring (EWSHM '14), Nantes, France, 2014.

[30] K. Prajapat and S. Ray-Chaudhuri, "Prediction error variances in Bayesian model updating employing data sensitivity," Journal of Engineering Mechanics, vol. 142, no. 12, 2016.

[31] K. Prajapat and S. Ray-Chaudhuri, "Posterior resolution and structural modification for parameter determination in Bayesian model updating," International Journal of Cybernetics and Informatics, vol. 5, no. 1, pp. 193-202, 2016. 


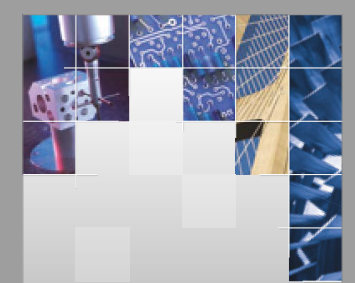

\section{Enfincering}
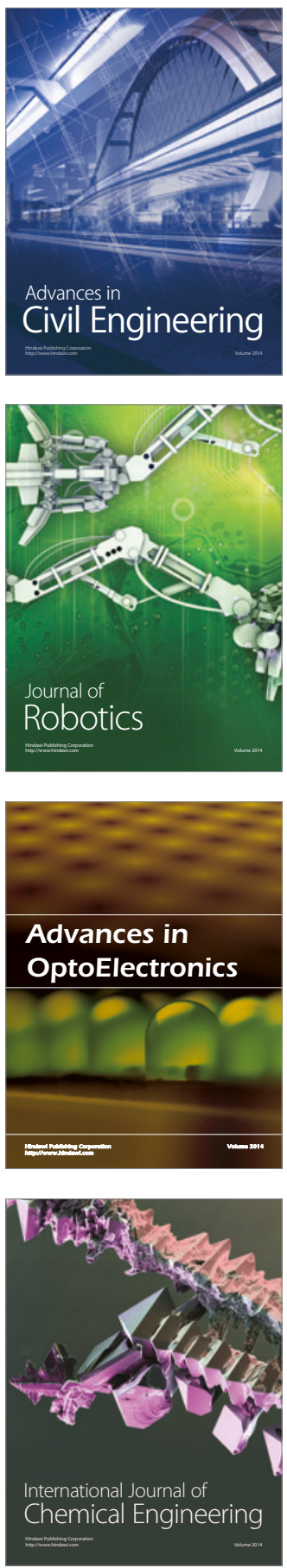

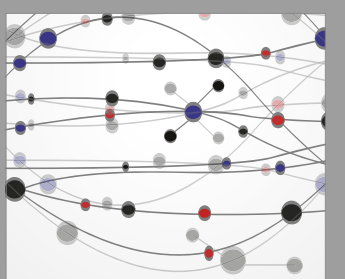

The Scientific World Journal

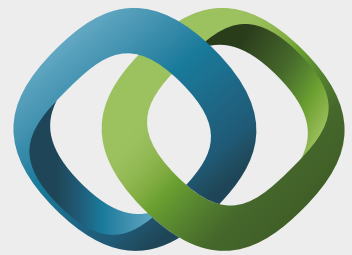

\section{Hindawi}

Submit your manuscripts at

https://www.hindawi.com
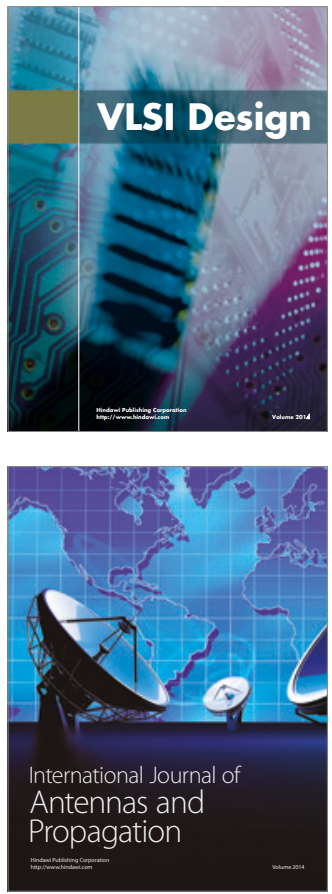

\section{Rotating}

Machinery
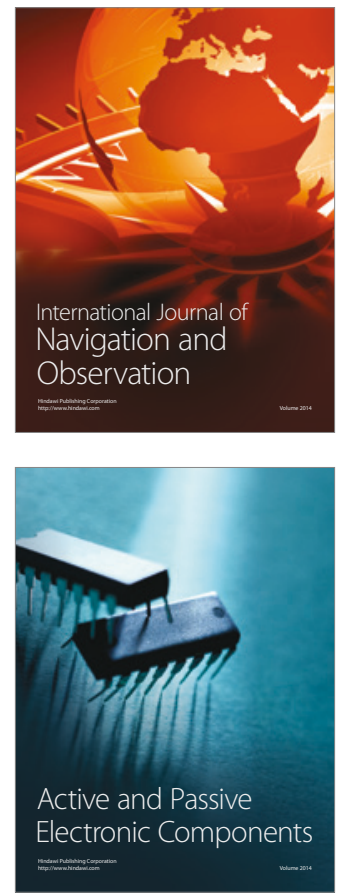
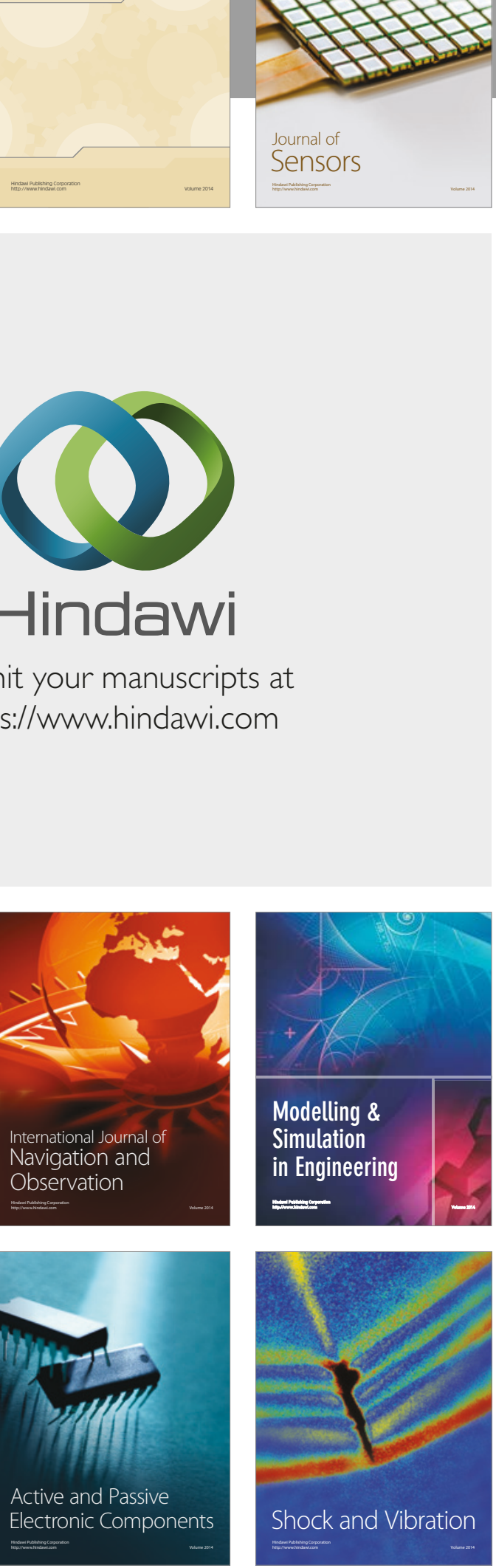
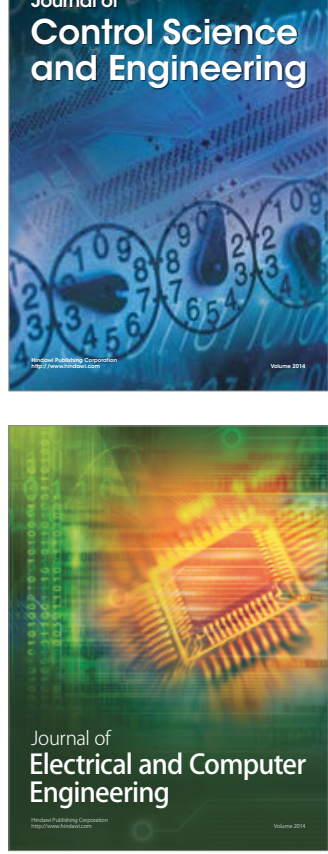

Distributed

Journal of

Control Science

and Engineering
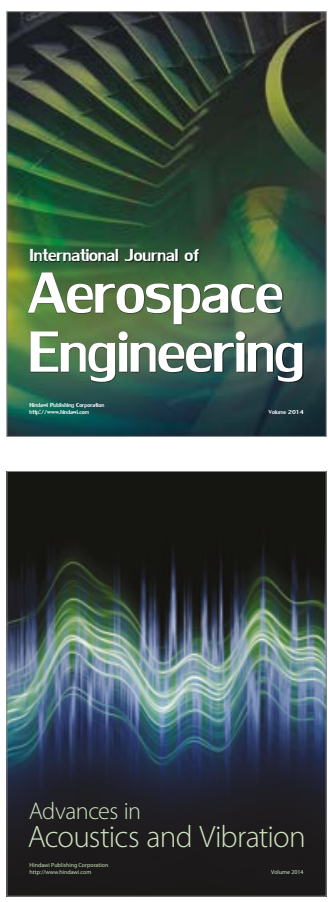

Sensor Networks 